\title{
Understanding therapies selected for managing pain associated with spinal cord injuries
}

\author{
Aneta A Surmanski
}

\section{ABSTRACT}

Pain is a common consequence in patients who are suffering from a spinal cord injury (SCI). Such pain affects nearly all aspects of life including mood, physical function, and social activities. Management of pain must be preceded with a thorough clinical examination which characterizes pain according to a classification scheme. The International Spinal Cord Injury Pain classification system divides pain into three tiers: according to type (nociceptive or neuropathic), according to subtype (localization of pain), and according to the source. Neuropathic pain is predominately treated with three different types of pharmaceutical therapies antidepressants, anticonvulsants, and analgesics. Multiple studies have shown amitriptyline, gabapentin, and pregabalin as being the most efficacious. Nociceptive pain is mainly treated with analgesics and physiotherapy, often together. Moreover, spasticity associated with nociceptive pain is treated with antispasmodics and physiotherapy. Non-traditional means of therapy, specifically transcutaneous electrical nerve stimulation, have demonstrated a favourable effect on pain. Nevertheless, finding an optimal treatment remains difficult and largely empirical. A stronger understanding of the mechanisms behind each pain type is needed in order to provide therapies that are better suited at targeting the source of the pain while minimizing possible side effects often seen in the SCI population. An integrated approach that fine tunes the combination of pharmacological and nonpharmacological therapies is needed for optimal management in SCI pain

\section{INTRODUCTION}

Pain associated with spinal cord injury (SCI) is a debilitating disorder that affects nearly $81 \%$ of individuals 1 year post-injury, and $82.7 \%$ of individuals 25 years post-injury. ${ }^{1}$ Chronic pain associated with SCI has been shown to not only affect daily activities, such as exercise and ability to work, but can also impact mental well-being and quality of life. ${ }^{2,3}$ A major challenge to those with SCI is that longterm prognosis of pain often worsens over time following injury, creating a difficult obstacle to recovery by contributing to disability and decreasing the patient's overall capacity in rehabilitation. ${ }^{1,3}$

Pain associated with spinal cord injury is classified according to the International Spinal Cord Injury Pain Classification and can be subdivided into two groups: neuropathic and nociceptive., ${ }^{1,4}$ Furthermore, neuropathic pain is further divided based on localization of pain: at-level, below-level or other, relative to site of injury, whereas nociceptive pain is divided into musculoskeletal, visceral or other pain. In addition, pain associated with spasticity is a problem often seen in SCI patients and may be managed using similar therapies as nociceptive pain. ${ }^{5}$

Treatment options vary for these subtypes of SCI pain and several guidelines, specifically CanPainSCI and Paralyzed Veterans of America (PVA), are available to guide physicians in recommending the appropriate course of treatment. These guidelines provide an in-depth analysis for screening, diagnosis and treatment for pain associated with SCI. ${ }^{6,7}$

Elucidating effective ways to alleviate pain remains an important factor in the recovery process, and understanding the specific mechanisms underlying these pain subtypes is vital in establishing proper efficacious treatment., ${ }^{2,34}$ Therefore, the objective of this review paper is to outline common mechanisms underlying pain subtypes in detail in order to describe how treatment options work to alleviate pain.

\section{NEUROPATHIC PAIN MANAGEMENT}

Neuropathic pain originates from the dysfunction or damage to the somatosensory system. ${ }^{4}$ This form of pain affects $29-75 \%$ of the SCI population. ${ }^{8}$ Etiology stems from structural damage to the neuron, which results in functional changes in neuronal signalling pathways, such as molecular changes in ion channels and Y-aminobutyric acid (GABA) metabolism. ${ }^{9}$ These changes may lead to neuronal hyperexcitability, permanent changes in nociceptive thresholds, and hypofunction of natural inhibitory signals. ${ }^{2,10}$ Hyperexcitability often results in the spontaneous generation of pain, and changes in nociceptive thresholds may pathologically amplify stimuli. ${ }^{11}$ Furthermore, natural inhibitory signals play a vital role in dampening or turning off specific signalling pathways. Disinhibition provokes neuronal excitation and spontaneous activity by prolonging synaptic transmission..$^{2,10-12}$ In addition, nerve injury may also cause reduced expression of opioid receptors, which in turn lowers sensitivity of those neurons to inhibition by opioid agonists. ${ }^{13}$

Pain location with respect to region of injury allows for subclassification of pain type and therefore allows physicians to recommend an appropriate course of treatment. ${ }^{2,8}$ Neuropathic pain at the level of injury is typically caused by nerve root compression and often results in numbness, pain, and tingling along the course of the nerve. Such pain is usually not related to the spinal cord itself but can be due to any number of etiologies, such as nerve root pathology or changes in supraspinal structures. ${ }^{14}$ Pain below the level of injury often originates from direct spinal cord trauma, causing changes in sensory function, but can also originate from other etiologies. ${ }^{4,5}$

Due to the complex nature of this chronic condition, neuropathic 
pain responds poorly to a single pharmaceutical drug. ${ }^{2,10}$ According to CanPainSCI guidelines, antidepressants, anticonvulsants, and analgesics are first-line pharmaceutical therapies used to treat neuropathic pain. $^{6}$ Currently, amitriptyline, pregabalin, and gabapentin have the best documented properties and should be prescribed first. ${ }^{2,415}$ Recent studies have shown a significant decrease in nerve pain experienced by patients treated with either pregabalin or gabapentin, compared to placebo. ${ }^{15-17}$ Gabapentin serves to reduce activity of voltage-gated calcium channels and increase GABA biosynthesis, both of which attenuate neuronal hyperexcitability. ${ }^{18}$ In addition, pregabalin also reduces neuronal hyperexcitability by increasing extracellular concentrations of GABA in the brain. ${ }^{19}$

Second-line treatment includes the use of opioids, specifically tramadol. ${ }^{6}$ These potent drugs may be used on their own or concurrently with other medications; ${ }^{20}$ however, risk of drug abuse and motion for side effects, such as gastrointestinal problems and cognitive deprivation, may complicate long-term use. Third-line therapy is transcranial direct current simulation, which shows a favourable effect on pain suppression. ${ }^{6}$ It modulates cortical excitability and decreases the perception of pain. ${ }^{21}$ Furthermore, evidence shows that transcutaneous electrical nerve stimulation either alone or with visual illusions and microsurgical lesioning of the dorsal root entry zone conferred neuropathic pain relief in patients that were resistant to other treatments., ${ }^{6,23,24}$ Both procedures are considered fourth-line therapy and are a last resort for patients experiencing neuropathic pain.

Although these treatment options convey promising outcomes, such therapies can impact other areas of function, specifically bowel and bladder management. For example, evidence shows that tricyclic antidepressants and opioids may cause constipation and increased urinary retention, among other side effects. ${ }^{25,26}$ Therefore, it is necessary to optimize these pharmacological treatments in individual patients in order to minimize these special side effects, while maximizing overall efficacy on pain management.

\section{NOCICEPTIVE PAIN MANAGEMENT}

Nociceptive pain is characterized as pain that follows when nonneural tissues, specifically musculoskeletal (MSK) tissues (bone, joint, muscle) are damaged. ${ }^{4}$ Patients in this category have a normal, functioning somatosensory nervous system but often experience mechanical instability, muscle trauma, and inflammation. ${ }^{11}$ MSK-related pain can originate from the initial trauma, postural abnormalities, and overuse of joints and muscles. ${ }^{27}$ Shoulder pain is the most common type and often results from overuse or muscle instability due to lack of core strength post-injury. ${ }^{28}$ Pain may also arise from spasticity, which affects muscle tone and contraction. ${ }^{29}$ Nociceptive pain can be further classified into visceral pain, which refers to pain generated in thoracic, abdominal, or pelvic viscera. ${ }^{3}$ Visceral structures are highly sensitive to a specific subset of stimuli, including stretch, inflammation, and ischemia, and generation of such stimuli may arise from bowel irregularities and side effects from certain medications. ${ }^{30}$
First-line therapy for nociceptive pain includes analgesics and physiotherapy. ${ }^{31}$ Analgesic medications, such as non-steroidal anti-inflammatory drugs and certain steroids, can help relieve pain caused by inflammation by blocking enzymes responsible for prostaglandin synthesis. ${ }^{32}$ On the other hand, physiotherapy strives to prevent and treat the overuse of muscles and joints by teaching patients how to properly stretch and increase strength, while attempting to maintain or regain motor tasks. ${ }^{33}$ Evidence shows that physical activity alone is capable of improving quality of movement, coordination, and flexibility but has also been shown to reduce depression, pain and stress. ${ }^{27,34}$ Moreover, physiotherapy can address poor posture, abnormal gait, and muscle overuse, which can all contribute to nociceptive pain., ${ }^{2,4}$ Second-line treatment consists of weak opioids, such as codeine, which help modulate pain by binding to receptors in the brain, spinal cord, and other nervous tissue responsible for analgesia. ${ }^{26}$ These therapies must be used with caution due to the risk of building tolerance and potential development of gastrointestinal problems. ${ }^{5,12,33}$

According to the PVA Guidelines for preservation of upper limb function post-SCI injury, MSK pain originating from upper extremities is managed using similar principles as in the nonSCI population. ${ }^{7}$ Healthcare providers must educate patients to minimize frequency and force of repetitive tasks as well as to avoid extreme positioning of the shoulder. Additionally, flexibility and resistance training should be integrated into the overall fitness program in order to enhance muscular endurance and promote appropriate limb positioning. ${ }^{7,28,35}$ On the other hand, visceral pain is treated based on underlying etiology, which may stem from distension of hollow organs and, most commonly, side effects from medications, i.e. constipation associated with opioid use. ${ }^{36}$ Treatment options include analgesics and spasmolytics. ${ }^{30,32}$

Spasticity is classified as the involuntary activation of muscles which often results in increased muscle tone and frequent phasic stretch reflexes. ${ }^{8,37}$ Such alterations in motor control lead to uncontrollable muscle spasms and pain and interfere with movement, gait, and speech. ${ }^{8}$ Treatment for spasticity is centered on treating spasticity itself or managing pain associated with spasticity. ${ }^{29,38}$ Antispasmodics, like baclofen, and physiotherapy represent the first-line treatment for spasticity itself. ${ }^{37-39}$ Baclofen works as an inhibitory neurotransmitter analog by blocking excitation pathways in the spinal cord, thereby promoting muscle relaxation. ${ }^{40}$

Lastly, non-traditional means of treatment, specifically cognitive-behavioural counselling and psychotherapy, have also been shown to provide significant advantages both on their own or in conjunction with more traditional therapies. ${ }^{41}$ Psychotherapy teaches patients how to develop better coping mechanisms to deal with both neuropathic and nociceptive pain as well as to decrease stress and improve overall quality of life. ${ }^{41,42}$

\section{CONCLUSION}

Pain associated with SCI is complex and requires a combination of treatment modalities, specifically pharmacological 
methods, physical therapy, and psychological counselling. Although individualizing treatment is very important, there are common considerations in the SCI population that are not present in other populations. These include impairment in bladder and bowel function, limited range of mobility, and impact on overall rehabilitation. ${ }^{20}$ With these careful considerations in mind and availability of more up-to-date guidelines, physicians can offer better combinational therapy options suited for each pain subtype associated with SCI.

\section{REFERENCES}

1. Cardenas DD, Jensen MP. Treatments for chronic pain in persons with spinal cord injury: a survey study. J Spinal Cord Med. 2006 Feb;29:109-17

2. Finnerup NB, Baastrup C. Spinal Cord Injury Pain: Mechanisms and Management. Curr Pain Headache Rep. 2012 Jun;16:207-16

3. Siddall PJ. Management of neuropathic pain following spinal cord injury: now and in the future. Spinal Cord. 2009 May;47:352-9

4. Hagen EM, Rekand T. Management of Neuropathic Pain Associated with Spinal Cord Injury. Pain Ther. 2015 Jun;4:51-65

5. Finnerup NB. Neuropathic pain and spasticity: intricate consequences of spinal cord injury. Spinal Cord. 2017 Jul;1-15

6. Loh E, Guy SD, Mehta S, et al. The CanPain SCI Clinical Practise Guidelines for Rehabilitation Management of Neuropathic Pain after Spinal Cord: introduction, methodology and recommendation overview. Spinal Cord. 2016 Jul;54:S1-6

7. Paralyzed Veterans of America Consortium for Spinal Cord Medicine. Preservation of upper limb function following spinal cord injury: a clinical practice guideline for health-care professionals. J Spinal Cord Med. 2005 Aug;28(5)434-70

8. Hama A, Sagen J. Combination Drug Therapy for Pain following Chronic Spinal Cord Injury. Pain Res \& Treatment. 2012 Mar;10:1-13

9. DeFrates S, Cook AM. Pharmacologic treatment of neuropathic pain following spinal cord injury. Orthopedics. 2011 Mar;34(3):203-7

10. Allison DJ, Thomas A, Beaudry K, et al. Targeting inflammation as a treatment modality for neuropathic pain in spinal cord injury: a randomized clinical trial. J of Neuroinflam. 2016 Jun;13:152-62

11. Costigan M, Scholz J, Woolf CJ. Neuropathic Pain: A maladaptive response of the nervous system to damage. Annu Rev Neurosci. 2009 May;32:1-32

12. Siddall PJ, Middleton JW. Spinal cord injury-induced pain: mechanisms and treatments. Pain Manag. 2015 Sept;5(6):493-507

13. Kohno T, Ji RR, Allchorne AJ, et al. Peripheral axonal injury results in reduced mu opioid receptor pre- and post-synaptic action in the spinal cord. Pain. 2005 Sept;117(1-2):77-87

14. Siddall P, Taylor DA, Cousins MJ. Classification of pain following spinal cord injury. Spinal Cord. 1997 Feb;35:69-75

15. Tai Q, Kirshblum S, Chen B, et al. Gabapentin in the treatment of neuropathic pain after spinal cord injury: a prospective, randomized, double-blind, crossover trial. J Spinal Cord Med. 2002 Jul;25(2):100-5.

16. Ahn SH, Park HW, Lee BS, et al. Gabapentin effect on neuropathic pain compared among patients with spinal cord injury and different durations of symptoms. Spine. 2003 Feb;28(4):341-6.

17. Mehta S, Mcintyre A, Dijkers M, et al. Gabapentinoids are effective in decreasing neuropathic pain and other secondary outcomes after spinal cord injury: a metaanalysis. Arch Phys Med Rehabil. 2014 Nov;95(11):2180-6

18. Taylor CP. Mechanisms of action of gabapentin. Rev Neurol. 1997 Feb;1:39-45

19. Taylor CP, Angelotti T, Faurman E. Pharmacology and mechanism of action of pregabalin: the calcium channel alpha2-delta (alpha2-delta) subunit as a target for antiepileptic drug discovery. Epilepsy Res. 2007 Feb;73(2):137-50

20. Mu A, Weinberg E, Moulin DE, et al. Pharmacologic management of chronic neuropathic pain: review of the Canadian pain society consensus statement. Can Fam Physician. 2017 Nov;63(11):844-52

21. Knotkova H, Cruciani RA. Non-invasive transcranial direct current simulation for the study and treatment of neuropathic pain. Methods Mol Bio. 2010 Feb;617:505-15

22. Mehta S, Orenczuk K, McIntyre A, et al. Neuropathic pain post spinal cord injury part 2: systematic review of dorsal root entry zone procedure. Top Spinal Cord Inj Rehabil. 2013 Jun;19(1):78-86.

23. Norrbrink C. Transcutaneous electrical nerve stimulation for treatment of spinal cord injury neuropathic pain. J Rehabil Res Dev. 2009 Jan;46(1): 85-93.

24. Celik EC, Erhan B, Gunduz B, et al. The effect of low-frequency TENS in the treatment of neuropathic pain in patients with spinal cord injury. Spinal Cord. 2013 Jan;51(4):334-7.

25. Remick RA. Anticholinergic side effects of tricyclic antidepressants and their management. Prog Neuropsychopharmacol Biol Psychiatry. 1988 Feb;12:225-31

26. Benyamin R, Trescot AM, Datta S, et al. Opioid complications and side effects. Pain Physician. 2008 Mar;11(2):105-20

27. Cooper L, Ells L, Ryan C, et al. Perceptions of adults with overweight/obesity and chronic musculoskeletal pain: an interpretative phenomenological analysis. J Clin Nurs 2017 Nov

28. Dalyan M, Cardenas DD, Gerard B. Upper extremity pain after spinal cord injury. Spinal Cord. 1999 Apr;37:191-5

29. Paolucci S, Martinuzzi A, Scivoletto G, et al. Assessing and treating pain associated with stroke, multiple sclerosis, cerebral palsy, spinal cord injury and spasticity. E J of Phys and Rehab Med. 2016 Aug;52(6): 827-40

30. Kogos Jr S, Richards JS, Banos JH, et al, Visceral pain and life quality in persons with spinal cord injury: a brief report. J Spinal Cord Med. 2005 Jan;28(3):333-7

31. Walters ET. Nociceptors as chronic drivers of pain and hyperreflexia after spinal cord injury: an adaptive-maladaptive hyperfunctional state hypothesis. Front in Physiol. 2012 Aug;3:309-22

32. Cashman JN. The mechanisms of action of NSAIDs in analgesia. Drugs. 1996 Nov;52(S5):13-23

33. Hadjipavlou G, Cortese AM, Ramaswamy B. Spinal cord injury and chronic pain. BJA Education. 2016 Aug;16(8):264-8

34. Focht BC, Koltn KF. Alterations in pain perception after resistance exercise performed in the morning and evening. J Strength Cond Res. 2009 May;23(3):891-7

35. Grant KL, Nagle KG, Cowan RE, et al. Body systems effects of a multi-modal training program targeting chronic, motor complete thoracic spinal cord injury. J of Neurotrauma. 2017 Aug;34:1-13

36. Wesselmann U, Baranowski A, Borjesson M, et al. Emerging therapies and novel approaches to visceral pain. Drug Discov Today Ther Strateg. 2009 Sept;6(3):89-95

37. Luo D, Wu G, Ji Y, et al. The comparative study of clinical efficacy and safety of baclofen vs tolperisone in spasticity caused by spinal cord injury. Saudi Pharm J. 2017 May;25:655-9

38. Patejdl R, Zettl UK. Spasticity in multiple sclerosis: contribution of inflammation, autoimmune mediated neuronal damage and therapeutic interventions. Autoimmunity Reviews. 2017 Sept;16:925-36

39. Perez-Arredondo A, Cazares-Ramirez E, Carrillo-Mora P, et al. Baclofen in the therapeutic of sequel of traumatic brain injury: spasticity. Clin Neuropharm. 2016 Jan;39:311-9

40. Milanov IG. Mechanisms of baclofen action on spasticity. Acta Neurol Scand. 1992 Jun;85(5):305-10

41. Shin JC, Kim NY, Chang SH, et al. Effect of Patient Education on Reducing Medication in Spinal Cord Injury Patients with Neuropathic Pain. Ann Rehabil Med. 2017 Aug;41(4):621-30

42. Burke D, Lennon O, Nolan M, et al. A cognitive behavioural therapy pain management programme for neuropathic pain post spinal cord injury: a feasibility study including the clinician and patient perspectives. Phys Med Rehabil Int. 2017 Aug;4(3):1119-29 